\title{
NONLINEAR ANALYSIS OF VEHICLE CONTROL ACTUATIONS BASED ON CONTROLLED INVARIANT SETS
}

\author{
BALÁZs NÉMETH ${ }^{a, *}$, PÉTER GÁSPÁR ${ }^{a}$, TAMÁS PÉNI ${ }^{a}$ \\ ${ }^{a}$ Systems and Control Laboratory, Institute for Computer Science and Control \\ Hungarian Academy of Sciences, Kende utca 13-17, 1111 Budapest, Hungary \\ e-mail: \{balazs.nemeth, peter.gaspar, tamas.peni\}@sztaki.mta.hu
}

\begin{abstract}
In the paper, an analysis method is applied to the lateral stabilization problem of vehicle systems. The aim is to find the largest state-space region in which the lateral stability of the vehicle can be guaranteed by the peak-bounded control input. In the analysis, the nonlinear polynomial sum-of-squares programming method is applied. A practical computation technique is developed to calculate the maximum controlled invariant set of the system. The method calculates the maximum controlled invariant sets of the steering and braking control systems at various velocities and road conditions. Illustration examples show that, depending on the environments, different vehicle dynamic regions can be reached and stabilized by these controllers. The results can be applied to the theoretical basis of their interventions into the vehicle control system.
\end{abstract}

Keywords: vehicle dynamics, sum-of-squares programming, Lyapunov method.

\section{Introduction and motivation}

In road vehicles, several active components are applied simultaneously to handle various performance requirements. Since, in their simultaneous operation, interference or conflicts may occur in the entire vehicle system, the coordination of the operations must be guaranteed. Integrated control is able to improve safety by modifying the operations of local controllers. If performance degradation has occurred in the operation of an actuator/sensor, the degraded element is substituted for by another one which provides similar dynamic effects. This coordination must be based on a theoretical method.

Recently, several important survey papers have been presented on the topic of integrated vehicle control (see, e.g., Yu et al., 2008; Cairano et al., 2013). Here are a few examples. A vehicle control with four-wheel-distributed steering and four-wheel-distributed traction/braking systems was proposed by Ono et al. (2006). A strategy with throttle control and automatic transmission was proposed by Kim et al. (2007). A yaw stability control system in which an active torque distribution and differential braking systems are used was put forward by Zhang et al. (2009). The integration of differential braking and front steering was proposed by Cairano

* Corresponding author et al. (2013). An integrated control that involves both four-wheel steering and yaw moment control was designed by Jianyong et al. (2007). Active steering and suspension controllers were also integrated to improve yaw and roll stability (Mastinu et al., 1994). A global chassis control involving an active/semi-active suspension and brake was proposed by Poussot-Vassal et al. (2008). A reconfigurable and fault-tolerant control system based on model predictive control (MPC) for the stop-and-go function was applied by Yetendje et al. (2012). Reliability estimation in degraded functional conditions relating to actuator faults was introduced by Khelassi et al. (2011). In integrated control systems the characteristics of the drivers' behavior were also taken into consideration in the control (Lu and Filev, 2009).

Based on physical considerations, an analytical method for the analysis of stability regions was provided by Pacejka (2004). Comprehensive studies on both system and structural stability were done by Sadri and Wu (2013). The control design focused only on steering control. The design of lateral stability control based on set-theoretical methods was proposed by Palmieri et al. (2011). In the control design an approximate piecewise-affine nonlinear dynamical model of the vehicle was applied (Palmieri et al., 2012; Carvalho et al., 2013). Control methods in which there were large operating regions accessible by the 
driver and smooth interventions at the stability boundaries were proposed by Kritayakirana and Gerdes (2012b), as well as Beal and Gerdes (2013).

Although vehicle control actuation selection has been usually performed using practical considerations, (see, e.g., Németh and Gáspár, 2011), in this paper, a theoretical basis for the coordination of control actuations is proposed. The stability regions of the maximum control inputs are also calculated. The aim of the analysis is to identify similarities and differences between the different control actuations. In an earlier paper, a reachable set-based analysis was presented to illustrate the benefits of the integration of the steering and brake controllers (see Németh and Gáspár, 2013). Although the reachable set analysis of linear vehicle models can be a relatively fast and easily applicable technique for determination of the actuator intervention limit, it has some drawbacks concerning the linear approach. In the paper, a nonlinear polynomial sum-of-squares (SOS) programming method is applied to calculate the shape of the controlled invariant sets of vehicle control actuations. A possible application of the analysis in the control strategy to guarantee vehicle stability is also proposed.

The SOS programming method is developed for general nonlinear systems. It can be widely used for systems whose nonlinearities are polynomial or can be approximated with polynomial functions. Since the tire force is described by a nonlinear function, the SOS method can be applied to various road vehicles. In the proposed method, the polynomial approximation of the tire characteristics provides an opportunity for handling the lateral vehicle dynamics.

Remark 1. In the paper, the definition of the global asymptotic stability is used. The system $\dot{x}=f(x)$ is asymptotically stable at $x=0$ if it is stable at $x=0$ and, additionally, there exists $h>0$ such that, if $\left\|x_{0}\right\|<h$, then $\lim _{t \rightarrow \infty}\left\|\phi_{t}\left(x_{0}\right)\right\|=0$. Furthermore, if $\forall x_{0} \in$ $\mathbb{R}^{n}, \lim _{t \rightarrow \infty}\left\|\phi_{t}\left(x_{0}\right)\right\|=0$, then the system is globally asymptotically stable (Jarvis-Wloszek, 2003).

The stability problem of road vehicles in the focus of the paper is that the oversteer of the vehicle during cornering is eliminated by reducing the side slip values of both the front and rear axles. The problem posed by the oversteer is that the vehicle may become dynamically unstable with a tendency to spin out due to a small lateral disturbance (e.g., wind forces). If the side slip values converge to zero, the vehicle can be steered either neutrally or with understeer during cornering. Note that stability can be guaranteed in an understeered vehicle.

In the paper, a polynomial state space representation of the model is used with two state variables, i.e., the side-slip angles of the front and rear axles $\alpha_{1}$ and $\alpha_{2}$. The dynamic stability of the system can be guaranteed if these state variables converge to zero. The computation method of controlled invariant sets proposed in the paper provides a domain of state variables in which the understeer can be guaranteed.

The contribution of the paper is the following. A three-step iterative method is developed for the computation of the maximum controlled invariant sets. The nonlinear tire characteristics are approximated by a polynomial form in a wide operation range; consequently, the stability analysis is valid in practical applications. Using the proposed method, the operations of the steering and brake actuations are analyzed and compared.

The paper is organized as follows. In Section 2 the nonlinear polynomial vehicle model and stability regions are formulated. The fundamentals of the SOS programming method used are detailed in Section 3 In Section 4, the computation method of maximum controlled invariant sets of polynomial lateral dynamical vehicle model is presented. The practical computation of the SOS problem is illustrated in an example. The computation results of the invariant sets at different velocities and adhesion coefficients are presented through simulation examples in Section 5 The control application of the set analysis is found in Section 6 . Section 7 contains some concluding remarks.

\section{Nonlinear modeling of lateral vehicle dynamics}

The starting point is a nonlinear lateral vehicle model, on which the analysis of the actuation efficiency is based (see Fig. 1). The two control actuations of the system are the differential braking torque $M_{b r}$ and the front wheel steering angle $\delta$. First, the formulation of the lateral dynamical model is detailed, which incorporates the nonlinearities of the tire characteristics. Second, the stability regions of the vehicle are illustrated based on a simulation example.

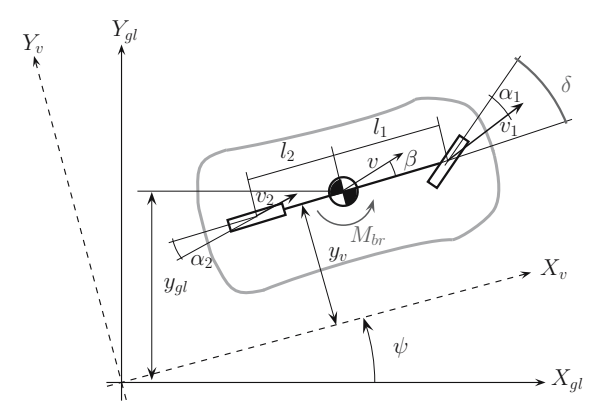

Fig. 1. Scheme of the lateral vehicle model. 
The torque $M_{b r}$ results from the different braking forces on the wheels. Although the control-oriented vehicle formulation is a single-track bicycle model, the control torque $M_{b r}$ can be actuated. In the implementation, the control input $M_{b r}$ is transformed into wheel forces, which are distributed between the four wheels. The force distribution must guarantee that the braking forces on the wheels generate $M_{b r}$.

2.1. Formulation of the vehicle model. Modeling tire forces is a crucial point of vehicle dynamics. Several tire models in which the nonlinearity of longitudinal and lateral tire forces is formed accurately have already been published (see, e.g., Pacejka, 2004; Kiencke and Nielsen, 2000; de Wit et al., 1995). In the paper, a polynomial tire modeling approach is presented, by which the nonlinearities of the tire characteristics are considered in a given operation range. The nonlinear characteristics of the lateral tire force in the function of tire side-slip $\alpha$ are illustrated in Fig. 2. The polynomial approximation is formulated as

$$
\mathcal{F}(\alpha)=\sum_{k=1}^{n} c_{k} \alpha^{k}=c_{1} \alpha+c_{2} \alpha^{2}+\cdots+c_{n} \alpha^{n} .
$$

In the example presented in Fig. 2, the exponent $n$ is chosen to be 10 . Using this approximation, the tire model is valid between $\alpha=-12^{\circ}$ and $\alpha=+12^{\circ}$. Note

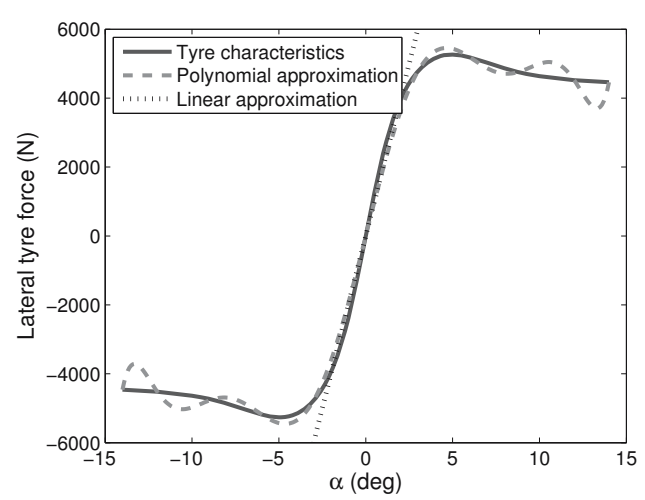

Fig. 2. Modeling of the lateral tire force.

that the tire characteristics are obtained from the vehicle simulation software CarSim.

The lateral dynamics of the vehicle are formulated by the following equations. The first one represents the dynamics of the yaw moment, while the second forms the dynamics of the lateral force:

$$
\begin{aligned}
J \ddot{\psi} & =\mathcal{F}_{1}\left(\alpha_{1}\right) l_{1}-\mathcal{F}_{2}\left(\alpha_{2}\right) l_{2}+M_{b r}, \\
m v(\dot{\psi}+\dot{\beta}) & =\mathcal{F}_{1}\left(\alpha_{1}\right)+\mathcal{F}_{2}\left(\alpha_{2}\right),
\end{aligned}
$$

where $m$ is the mass of the vehicle, $J$ is yaw-inertia, $l_{1}$ and $l_{2}$ are geometric parameters, $\beta$ is the side-slip angle of the chassis, $\dot{\psi}$ is the yaw-rate. $\mathcal{F}_{1}\left(\alpha_{1}\right)$ and $\mathcal{F}_{2}\left(\alpha_{2}\right)$ represent lateral tire forces, which depend on tire side-slip angles $\alpha_{1}$ and $\alpha_{2}$. The relationships between the tire side-slip angles for the front and rear axles, the steering angle of the vehicle and the side-slip angle of the chassis are $\tan \left(\delta-\alpha_{1}\right)=\left(l_{1} \dot{\psi}+v \sin \beta\right) /(v \cos \beta), \tan \left(\alpha_{2}\right)=$ $\left(l_{2} \dot{\psi}-v \sin \beta\right) /(v \cos \beta)$. In stable driving conditions tire side-slip angle $\alpha_{i}$ is normally no greater than $12^{\circ}$ and the equations can be simplified by substituting $\sin \beta \approx \beta$ and $\cos \beta \approx 1$. Moreover, the relative error of these simplifications is less than $1 \%$ (see also Kritayakirana and Gerdes, 2012a). Thus, we get the following side-slip angles of the front and rear axles:

$$
\begin{aligned}
& \alpha_{1}=\delta-\beta-\frac{\dot{\psi} l_{1}}{v}, \\
& \alpha_{2}=-\beta+\frac{\dot{\psi} l_{2}}{v} .
\end{aligned}
$$

In the following, (3) is used to transform (2) into a polynomial state-space representation $\dot{x}=f(x)+g u$, where $x$ is the state vector, $u$ is the control input signal, $f$ and $g$ are vectors.

Remark 2. In several control applications, the lateral forces are approximated with linear functions, such as $\mathcal{F}_{i}\left(\alpha_{i}\right)=c_{i} \alpha_{i}, \quad i=\{1,2\}$, where $c_{i}$ is cornering stiffness. The advantage of this formulation is the simple description, although the linear tire model can be used in a narrow tire side-slip range $\left( \pm 3^{\circ}\right)$ (see Fig. 2).

The yaw rate and the side slip of the vehicle can be expressed from (3) in the following forms:

$$
\begin{aligned}
& \dot{\psi}=v \frac{\alpha_{2}-\alpha_{1}+\delta}{l_{1}+l_{2}}, \\
& \beta=-\frac{\alpha_{1} l_{2}+\alpha_{2} l_{1}-l_{2} \delta}{l_{1}+l_{2}} .
\end{aligned}
$$

Equation (2) contains the time derivatives of $\dot{\psi}$ and $\beta$, which must be differentiated to obtain $\ddot{\psi}$ and $\dot{\beta}$. At a constant velocity $v$, the derivatives are

$$
\begin{aligned}
& \ddot{\psi}=v \frac{\dot{\alpha}_{2}-\dot{\alpha}_{1}+\dot{\delta}}{l_{1}+l_{2}} \\
& \dot{\beta}=-\frac{\dot{\alpha}_{1} l_{2}+\dot{\alpha}_{2} l_{1}-l_{2} \dot{\delta}}{l_{1}+l_{2}} .
\end{aligned}
$$

Now the vehicle model (2) is reformulated using (4) and (5): 


$$
\begin{aligned}
\dot{\alpha}_{2}-\dot{\alpha}_{1}= & {\left[\frac{l_{1}+l_{2}}{J v}\left(\mathcal{F}_{1}\left(\alpha_{1}\right) l_{1}-\mathcal{F}_{2}\left(\alpha_{2}\right) l_{2}\right)\right] } \\
& -\dot{\delta}+\frac{l_{1}+l_{2}}{J v} M_{b r}, \\
\dot{\alpha}_{1} l_{2}+\dot{\alpha}_{2} l_{1}= & v\left(\alpha_{2}-\alpha_{1}\right)-\frac{l_{1}+l_{2}}{m v}\left[\mathcal{F}_{1}\left(\alpha_{1}\right)+\mathcal{F}_{2}\left(\alpha_{2}\right)\right] \\
& +v \delta+l_{2} \dot{\delta} .
\end{aligned}
$$

The rearrangement of the vehicle model shows that the new states of the model are tire slip angles $\alpha_{1}$ and $\alpha_{2}$. In this way, the nonlinearity of the lateral tire forces $\mathcal{F}_{1}, \mathcal{F}_{2}$ can be considered. However, 6 includes the time derivative of the front-wheel steering angle. Since $\delta$ is a control input, $\dot{\delta}$ is modeled as

$$
\dot{\delta} \cong \max \left(\frac{|\dot{\delta}|}{|\delta|}\right) \cdot \delta=\nu \cdot \delta
$$

where parameter $\nu$ represents the relationship between the maximum steering value and the variation speed of $\delta$. Since $\max \delta$ is a given fixed limit in the actuation analysis, a high $\nu$ value represents a fast-changing steering signal, while a slow-changing steering signal is modeled with low $\nu$.

Remark 3. Németh and Gáspár (2011) proposed a second-order steering model, by which $\delta$ and $\dot{\delta}$ are regarded as system states. In this case, the control input of the system is the steering torque generated by an electric motor or by the driver. Although the application of the proposed steering model leads to a more sophisticated lateral vehicle model, it increases the complexity of the polynomial one. Thus the simplified modeling approach (7) is applied in the following analysis.

The polynomial state-space representation of the system is formulated using (6) and the substitution of (7) is as follows:

$$
\dot{x}=\left[\begin{array}{c}
\dot{\alpha}_{1} \\
\dot{\alpha}_{2}
\end{array}\right]=\left[\begin{array}{c}
\mathrm{f}_{1}\left(\alpha_{1}, \alpha_{2}\right) \\
\mathrm{f}_{2}\left(\alpha_{1}, \alpha_{2}\right)
\end{array}\right]+\left[\begin{array}{l}
g_{1} \\
g_{2}
\end{array}\right] M_{b r}+\left[\begin{array}{c}
h_{1} \\
h_{2}
\end{array}\right] \delta,
$$

where

$$
\begin{aligned}
\mathrm{f}_{1}= & \frac{l_{1}}{J v}\left[\mathcal{F}_{2}\left(\alpha_{2}\right) l_{2}-\mathcal{F}_{1}\left(\alpha_{1}\right) l_{1}\right] \\
& +\frac{v}{l_{1}+l_{2}}\left(\alpha_{2}-\alpha_{1}\right)-\frac{1}{m v}\left[\mathcal{F}_{1}\left(\alpha_{1}\right)+\mathcal{F}_{2}\left(\alpha_{2}\right)\right], \\
\mathrm{f}_{2}= & \frac{l_{2}}{J v}\left[\mathcal{F}_{1}\left(\alpha_{1}\right) l_{1}-\mathcal{F}_{2}\left(\alpha_{2}\right) l_{2}\right] \\
& +\frac{v}{l_{1}+l_{2}}\left(\alpha_{2}-\alpha_{1}\right)-\frac{1}{m v}\left[\mathcal{F}_{1}\left(\alpha_{1}\right)+\mathcal{F}_{2}\left(\alpha_{2}\right)\right], \\
& h_{1}=\frac{v}{l_{1}+l_{2}}+\nu, \quad h_{2}=\frac{v}{l_{1}+l_{2}}, \\
g_{1}=-\frac{l_{1}}{J v}, & g_{2}=\frac{l_{2}}{J v} .
\end{aligned}
$$

The proposed vehicle model 8 contains a differential braking torque and front wheel steering. In the forthcoming study, the system is analyzed using the control actuations separately. In the steering analysis, $M_{b r} \equiv 0$. In the examination of braking, $\delta \equiv 0$, which has an effect on the definition of the front tire side-slip $\alpha_{1}$ (see (3)).

2.2. Illustration of stability regions based on the vehicle model. In this section, the stability regions of the vehicle model are illustrated based on simulation examples. The vehicle parameters are found in Section 5 Figure 3 shows an illustration of the effect of polynomial tire force approximation on lateral vehicle modeling. In the corresponding figures, simulation results of the system $\dot{x}=f(x)$ with $x(0)=\left[\begin{array}{ll}\alpha_{1}(0) & \alpha_{2}(0)\end{array}\right]^{T}$ are shown. The initial slips are chosen in the interval $\alpha_{i}(0)=\left[-25^{\circ}, 25^{\circ}\right]$ using the step $2.5^{\circ}$. Figure 3(a) illustrates the result of the simulations with the original tire force characteristics, while Fig. 3(b) presents the result when polynomial approximation is used. Consequently, $2 \cdot 21 \cdot 21$ simulation scenarios are used in the simulation. The velocity in the vehicle model is constant, $v=40 \mathrm{~m} / \mathrm{s}$, during the simulations. Figure 3 shows the phase portrait of the autonomous system $\dot{x}=f(x)$, which is (8) with $\delta \equiv 0$, $M_{b r} \equiv 0$. The black lines illustrate the state trajectories which do not converge to zero. Simultaneously, the light-gray lines are the state trajectories which converge to zero. This means that the light-gray lines depict the initial states, where the system is stable.

It can be seen that the stable regions are close to each other in the two models in the validity region of the polynomial model $\left(-12^{\circ},+12^{\circ}\right)$. Outside the stable region, the two models are different: in the original model the system is unstable (see Fig. 3(a) , while in the polynomial model the system has two locally stable states (see Fig. 3(b) . Since the two locally stable points are outside the model validity region, they are indifferent in terms of the model applicability. The directions of the lines in the portrait are almost the same as in the validity region. Based on the analysis, it can be stated that the polynomial approximation results in almost the same stable and unstable regions when $\alpha_{i} \leq 12^{\circ}$. Since the stable regions are limited and rather small in the model validity region, it is necessary to find further states which can be stabilized by an appropriate peak-bounded control input signal $u$.

From the comparison of the two figures it can be seen that the polynomial approximation results in almost the same stable and unstable regions when $\alpha_{i} \leq 12^{\circ}$. In this region, polynomial fitting is sufficient and can be used for further analysis. Note that, although one example is shown in the paper, these simulations are performed at several velocities and $\mu$ scenarios. 


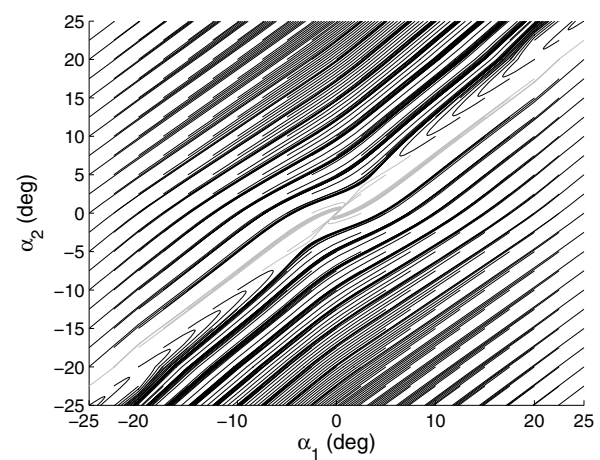

(a)

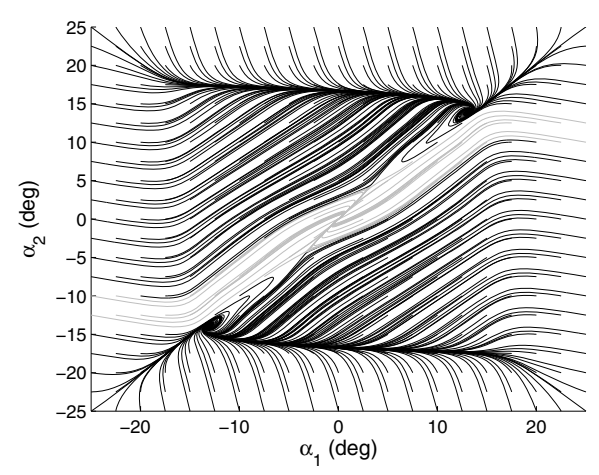

(b)

Fig. 3. Comparison of vehicle models: original model (a), polynomial model (b).

\section{Fundamentals of the SOS programming technique}

In this section, fundamental concepts concerning the SOS programming method are summarized. The method is suitable for analyzing and controling nonlinear polynomial systems.

Several papers deal with SOS programming, which has been elaborated on in the recent decade for control purposes. It is an efficient tool for finding feasible solutions to polynomial inequalities. In SOS programming, this problem is transformed into a semi-definite optimization task. Important theorems in SOS programming, such as the application of Positivstellensatz, were proposed by Parrilo (2003). In this way, the convex optimization methods can be used to find appropriate polynomials for the SOS problem. The approximation of nonnegative polynomials by a sequence of SOS was presented by Lasserre (2007). The SOS polynomials incorporate the original nonnegative polynomials in an explicit form.

Prajna et al. (2004) showed sufficient conditions for the solutions to nonlinear control problems, which were formulated in terms of state dependent linear matrix inequalities (LMIs). In the paper, the semidefinite programming relaxations based on the SOS decomposition were then used to efficiently solve such inequalities. The application of the SOS decomposition technique to non-polynomial system analysis was summarized by Papachristodoulou and Prajna (2005). Jarvis-Wloszek et al. (2003) introduced the application of SOS programming to several control problems, e.g., reachable set computation and control design algorithms. A local stability analysis of polynomial systems and an iterative computation method for their region of attraction were presented by Tan and Packard (2008). In the work of Scherer and Hol (2006), the SOS method was applied to two non-convex problems, for example, polynomial semi-definite programming and the fixed-order $\mathcal{H}_{2}$ synthesis problem.

Summers et al. (2003) presented performance analysis of polynomial systems by which sufficient conditions were provided for bounds on reachable sets and $\mathcal{L}_{2}$ gain of nonlinear systems subject to norm-bounded disturbance inputs. Robust performance in polynomial control systems was analyzed by Topcu and Packard (2009). That paper considered the effects of neglected dynamics and parametric uncertainties. Numerical computation problems of convex programming based on the SOS method in practical applications were analyzed by Löfberg (2009). As a new result, the maximum controlled invariant sets of polynomial control systems were calculated by Korda et al. (2013).

The following definitions and theorems are essential to understand SOS programming (Jarvis-Wloszek et al., 2003). Let $\mathbb{R}$ denote the set of real numbers and $\mathbb{Z}_{+}^{n}$ denote the set of nonnegative integers. The basic elements of the method are polynomials and SOS as defined below.

Definition 1. A polynomial $f$ in $n$ variables is a finite linear combination of the functions $m_{\alpha}(x):=x^{\alpha}=$ $x_{1}^{\alpha_{1}} x_{2}^{\alpha_{2}} \cdots x_{n}^{\alpha_{n}}$ for $\alpha \in \mathbb{Z}_{+}^{n}$, deg $m_{\alpha}=\sum_{i=1}^{n} \alpha_{i}$ :

$$
f:=\sum_{\alpha} c_{\alpha} m_{\alpha}=\sum_{\alpha} c_{\alpha} x^{\alpha}
$$

with $c_{\alpha} \in \mathbb{R}$. Define $\mathcal{R}_{n}$ to be the set of all polynomials in $n$ variables. The degree of $f$ is defined as $f:=$ $\max _{\alpha} \operatorname{deg} m_{\alpha}$.

Definition 2. The set of SOS polynomials in $n$ variables is defined as

$$
\Sigma_{n}:=\left\{p \in \mathcal{R}_{n} \mid p=\sum_{i=1}^{t} f_{i}^{2}, f_{i} \in \mathcal{R}_{n}, i=1, \ldots, t\right\}
$$

for some $t<\infty$

A central theorem of SOS programming is Positivstellensatz. By the application of this theorem, the set emptiness constraints of an optimization task can be transformed into SOS feasibility problems. 
Theorem 1. (Positivstellensatz) Given polynomials $\left\{f_{1}, \ldots, f_{r}\right\},\left\{g_{1}, \ldots, g_{t}\right\}$ and $\left\{h_{1}, \ldots, h_{u}\right\}$ in $\mathcal{R}_{n}$, the following are equivalent:

1. The set

$$
\left\{\begin{array}{l|l}
x \in \mathbb{R}^{n} & \begin{array}{c}
f_{1}(x) \geq 0, \ldots, f_{r}(x) \geq 0 \\
g_{1}(x) \neq 0, \ldots, g_{t}(x) \neq 0 \\
h_{1}(x)=0, \ldots, h_{u}(x)=0
\end{array}
\end{array}\right\}
$$

is empty.

2. There exists polynomials $f \in \mathcal{P}\left(f_{1}, \ldots, f_{r}\right)$ ( $\mathcal{P}$ is a multiplicative convex cone), $g \in \mathcal{M}\left(g_{1}, \ldots, g_{t}\right)(\mathcal{M}$ is a multiplicative monoid), $h \in \mathcal{I}\left(h_{1}, \ldots, h_{u}\right)(\mathcal{I}$ is ideal) such that

$$
f+g^{2}+h=0 .
$$

There is an important connection between SOS programming and LMI problems, which was proved by Parrilo (2003).

Theorem 2. (LMI feasibility problem) Given a finite set

$$
\left\{p_{i}\right\}_{i=0}^{m} \in \mathcal{R}_{n}
$$

the existence of $\left\{a_{i}\right\}_{i=0}^{m} \in \mathbb{R}_{n}$ such that

$$
p_{0}+\sum_{i=1}^{m} a_{i} p_{i} \in \Sigma_{n}
$$

is an LMI feasibility problem.

The previous two theorems can be used to prove a generalization of the S-procedure, which is highly significant in the forthcoming computations.

Theorem 3. (Generalized S-procedure) Given symmetric matrices $\left\{p_{i}\right\}_{i=0}^{m} \in \mathcal{R}_{n}$, if there exist nonnegative scalars $\left\{s_{i}\right\}_{i=1}^{m} \in \Sigma_{n}$ such that

$$
p_{0}-\sum_{i=1}^{m} s_{i} p_{i} \succeq q
$$

with $q \in \Sigma_{n}$, then

$$
\bigcap_{i=1}^{m}\left\{x \in \mathbb{R}^{n} \mid p_{i}(x) \geq 0\right\} \subseteq\left\{x \in \mathbb{R}^{n} \mid p_{0}(x) \geq 0\right\} .
$$

The related set emptiness question asks if

$$
\begin{array}{r}
W:=\left\{x \in \mathbb{R}^{n} \mid p_{1}(x) \geq 0, \ldots, p_{m}(x) \geq 0,\right. \\
\left.-p_{0}(x) \geq 0, p_{0}(x) \neq 0\right\}
\end{array}
$$

is empty.

\section{Computation method of controlled invariant sets}

A vehicle model with a polynomial tire model is formulated in Section 2 and the fundamentals of SOS programming for the polynomial model analysis are proposed in Section 3. In this section, the controlled invariant sets of the system are computed based on the preliminaries introduced.

The purpose of the nonlinear control actuation analysis is the determination of intervention limits of control actuation. In Section 2.1, it is shown that the vehicle has a limited stability region. With an appropriate intervention, some of the unstable regions can be stabilized. In the next section, an answer to the following question is sought: What is the largest state-space region in which the stability of the system can be guaranteed by a given peak-bounded control input? This question leads to the computation of controlled invariant sets (Korda et al., 2013).

4.1. Theoretical background. The state-space representation of the system is given in the following form (see (8)):

$$
\dot{x}=f(x)+g u,
$$

where $f(x)$ is a vector which incorporates smooth polynomial functions and $f(0)=0$. In the next analysis, one control input is considered, so that $u=M_{b r}$ or $u=\delta$. The global asymptotical stability of the system at the origin is guaranteed by the existence of the control Lyapunov function of the system defined as follows (Sontag, 1989)

Definition 3. A smooth, proper and positive-definite function $V: \mathbb{R}^{n} \rightarrow \mathbb{R}$ is a control Lyapunov function for the system if

$$
\inf _{u \in \mathbb{R}}\left\{\frac{\partial V}{\partial x} f(x)+\frac{\partial V}{\partial x} g \cdot u\right\}<0
$$

for each $x \neq 0$.

According to Definition 3 , at the candidate $V$, two main cases must be distinguished:

1: If $\frac{\partial V}{\partial x} f(x)<0$, then the system is stable and $u \equiv 0$. This stability scenario is contained by the next two stability criteria.

2: If $\frac{\partial V}{\partial x} f(x)>0$, then the system is unstable. However, the system can be stabilized:

2(a): $\frac{\partial V}{\partial x} g<0$ and $\frac{\partial V}{\partial x} f(x)+\frac{\partial V}{\partial x} g \cdot u_{\max }<0$. In this case, the upper peak-bound of control input $u$ stabilizes the system. 
2(b): $\frac{\partial V}{\partial x} g>0$ and $\frac{\partial V}{\partial x} f(x)-\frac{\partial V}{\partial x} g \cdot u_{\max }<0$. In this case, the lower peak-bound of control input $u$ stabilizes the system. Note that $u_{\min }=-u_{\max }$ is assumed.

The controlled invariant set of the system 17 is defined as the level-set of the control Lyapunov function at $V(x)=1$. Thus, the fulfilment of the previous stability criterion must be guaranteed at $V(x) \leq 1$.

Moreover, the Positivstellensatz and generalized S-procedure theorems (see Section 3) require nonstrict inequality $(\geq)$ conditions to formulate SOS conditions. Thus, the condition $\frac{\partial V}{\partial x} g<0$ in 2(a) is rewritten as $\frac{\partial V}{\partial x} g \leq-\epsilon$, where $\epsilon \in \mathbb{R}^{+}$is as small as possible. Similarly, in 2(b) $\frac{\partial V}{\partial x} g \geq \epsilon$ is written. Additionally, the conditions $\frac{\partial V}{\partial x} f(x) \pm \frac{\partial V}{\partial x} g \cdot u_{\max }<0$ in 2(a) and 2(b) are also reformulated to two conditions: $\frac{\partial V}{\partial x} f(x) \pm \frac{\partial V}{\partial x} g$. $u_{\max } \leq 0$ and $\frac{\partial V}{\partial x} f(x) \pm \frac{\partial V}{\partial x} g \cdot u_{\max } \neq 0$.

Above, the stability criterion of the polynomial system has been formed. Based on these constraints, it is necessary to find a control Lyapunov function $V$ which meets the following set emptiness conditions:

$$
\begin{aligned}
& \left\{-\frac{\partial V}{\partial x} g-\epsilon \geq 0,1-V(x) \geq 0, L_{1}(x) \neq 0,\right. \\
& \frac{\partial V}{\partial x} f(x)+\frac{\partial V}{\partial x} g u_{\max } \geq 0, \\
& \left.\frac{\partial V}{\partial x} f(x)+\frac{\partial V}{\partial x} g u_{\max } \neq 0\right\}=\emptyset, \\
& \left\{\frac{\partial V}{\partial x} g-\epsilon \geq 0,1-V(x) \geq 0, L_{2}(x) \neq 0,\right. \\
& \frac{\partial V}{\partial x} f(x)-\frac{\partial V}{\partial x} g u_{\max } \geq 0, \\
& \left.\frac{\partial V}{\partial x} f(x)-\frac{\partial V}{\partial x} g u_{\max } \neq 0\right\}=\emptyset \text {. }
\end{aligned}
$$

Note that the relations in the third inequality are inverted to guarantee the emptiness of the sets. The role of $L_{1,2}(x) \neq 0$ is to guarantee the condition $x \neq 0$ in Definition $3 . L_{1,2}(x)$ is chosen as a positive definite polynomial (Jarvis-Wloszek et al., 2003). Since it is necessary to find the maximum controlled invariant set, another set emptiness condition is also defined to improve the efficiency of the method (Jarvis-Wloszek et al., 2003):

$$
\{p(x) \leq \beta, V(x) \geq 1, V(x) \neq 1\}=\emptyset,
$$

where $p \in \Sigma_{n}$ is a fixed and positive definite function. $\beta$ defines a $P_{\beta}:=\left\{x \in \mathbb{R}^{n} \mid p(x) \leq \beta\right\}$ level set, which is incorporated in the actual controlled invariant set. Thus, the maximization of $\beta$ enlarges $P_{\beta}$ together with the controlled invariant set.

The set emptiness conditions are reformulated to SOS conditions based on the S-procedure (see Section 3).
Thus, the next optimization problem is formed to find the maximum controlled invariant set:

$$
\max \beta
$$

over SOS polynomials $s_{1}, s_{2}, s_{3}, s_{4}, s_{5} \in \Sigma_{n}$ and polynomials $V, p_{1}, p_{2} \in \mathcal{R}_{n}, V(0)=0$ such that

$$
\begin{aligned}
& -\left(\frac{\partial V}{\partial x} f(x)+\frac{\partial V}{\partial x} g u_{\max }\right)-s_{1}\left(-\frac{\partial V}{\partial x} g-\epsilon\right) \\
& -s_{2}(1-V)-p_{1} L_{1} \in \Sigma_{n}, \\
& -\left(\frac{\partial V}{\partial x} f(x)-\frac{\partial V}{\partial x} g u_{\max }\right)-s_{3}\left(\frac{\partial V}{\partial x} g-\epsilon\right) \\
& -s_{4}(1-V)-p_{2} L_{2} \in \Sigma_{n}, \\
& -\left(s_{5}(\beta-p)+(V-1)\right) \in \Sigma_{n} .
\end{aligned}
$$

Remark 4. The derivation of (22) resembles the one by Jarvis-Woszlek et al. (2013), but it is more complex because the cone is generated by three terms and there are two polynomials constrained to zero. Some terms of SOS conditions are omitted in the application of the Positivstellensatz. Although these conditions introduce conservatism, the size of the complexity of the numerical problem is reduced.

\subsection{Practical computation of the maximum con-} trolled invariant set. The optimization method of the maximum controlled invariant set has been proposed in the previous parts of the section. Although (22) provides an appropriate solution to the optimization problem, it results in numerical difficulties. Note that the degree of $f(x)$ is determined by the degree of the lateral tire model (see (1)).

A polynomial in $\theta$ variables of degree $2 N$ can be transformed into an LMI with $\left(\begin{array}{c}\theta+N \\ N\end{array}\right) \times\left(\begin{array}{c}\theta+N \\ N\end{array}\right)$ dimensions (see Parrilo, 2003). In the presented example (Fig. 2), the degree of the tire model is $2 N=10$, and the system has two variables: $\alpha_{1}$ and $\alpha_{2}$, thus $\theta=2$. The size of the LMI is $\left(\begin{array}{c}2+5 \\ 5\end{array}\right)^{2}=42 \cdot 42=1764$, which denotes LMI dimensions. Due to the vast size of the LMI feasibility task, numerical problems may occur. Therefore the resulting control Lyapunov function $V$ of optimization (22) must be checked.

In the following, an alternative computation method is proposed to find the maximum controlled invariant set, which, according to our experience, may lead to an easier calculation. The practical method contains a three-step iterative method.

Step 1. The region of attraction of the uncontrolled system $\dot{x}=f(x)$ is determined as an initial set. In this step, the maximum level set of $V_{0}=1$ is found, which is incorporated in the stable region. The SOS based computation of the region of attraction is presented by Jarvis-Wloszek (2003). 
Step 2. An $\eta$ parameter is chosen and $V_{\eta}=V_{0} \cdot \eta$ is checked as a local control Lyapunov function. The level-set $V_{\eta}=1$ represents a controlled invariant set $S_{\eta}$, in which the system can be stabilized using a finite control input $u$. Depending on parameter $\eta$, the size of the level-set can be enlarged or reduced. The SOS based computation of the local control Lyapunov function is proposed by Tan and Packard (2008). This step is in relation with (22) if $V$ is fixed and $u$ is not constrained.

Step 3. In the final step, the acceptability and the enlarging possibility of controlled invariant set $S_{\eta}$ must be checked. The peak-bounds of the actuation are $u_{\min }=-u_{\max }$ and $u_{\text {max }} \cdot S_{\text {unst }}=\frac{\partial V_{0}}{\partial x} f(x)>0$ is the unstable region of the system. $S_{\min }=\frac{\partial V_{0}}{\partial x} f(x)-\frac{\partial V_{0}}{\partial x} g \cdot u_{\max }>0$ is the region which cannot be stabilized by $u_{\min }$. Similarly, $S_{\max }=$ $\frac{\partial V_{0}}{\partial x} f(x)+\frac{\partial V_{0}}{\partial x} g \cdot u_{\max }>0$ is the region which cannot be stabilized by $u_{\max }$. If $S_{\eta}$ is an appropriate controlled invariant set and $V_{\eta}$ is an appropriate control Lyapunov function, then

$$
S_{\eta} \cap S_{\text {unst }} \cap S_{\min } \cap S_{\max }=\emptyset .
$$

The emptiness of the intersection condition defined above can be checked visually by plotting $S_{\eta}, S_{\text {unst }}, S_{\min }$ and $S_{\max }$. Additionally, if $S_{\eta}$ is appropriate, then $\eta$ can be reduced in the previous step to maximize the controlled invariant set.

Remark 5. The shape of the maximum controlled invariant set is fundamentally determined by the chosen $V_{0}$. If the result of the iterative method $V_{\eta}$ is not acceptable, the function $V_{0}$ of Step 1 should be modified.

The iterative determination of the maximum controlled invariant set is illustrated in the example presented in Section 2.2 The maximum set of the region of attraction is shown in Fig. 4 In this phase portrait, the light-gray regions are the open-loop stable regions, the black regions are locally stable $x_{\infty} \neq\left[\begin{array}{cc}0 & 0\end{array}\right]^{T}$, and the black set is the region of attraction. This bounding is a conservative approximation, which can be used as an initial set.

In Fig. 5 the sets $S_{\eta}, S_{\text {unst }}, S_{\min }$ and $S_{\max }$ of the controlled system are illustrated. The enlargement of $S_{\eta}$ is limited in the positive $\alpha_{2}$ regions by $S_{\min }$, in the negative $\alpha_{2}$ regions by $S_{\max }$.

\section{Maximum controlled invariant sets of vehicle control actuation}

In this section the computation results of the maximum controlled invariant sets are presented. The vehicle parameters are $m=1833 \mathrm{~kg}, J=2765 \mathrm{kgm}^{2}, l_{1}=$ $1.402 \mathrm{~m}, l_{2}=1.646 \mathrm{~m}$. The actuation limits are calculated in two tire-road adhesion coefficients $\mu=1$ (dry asphalt road) and $\mu=0.4$ (wet asphalt road). The tire

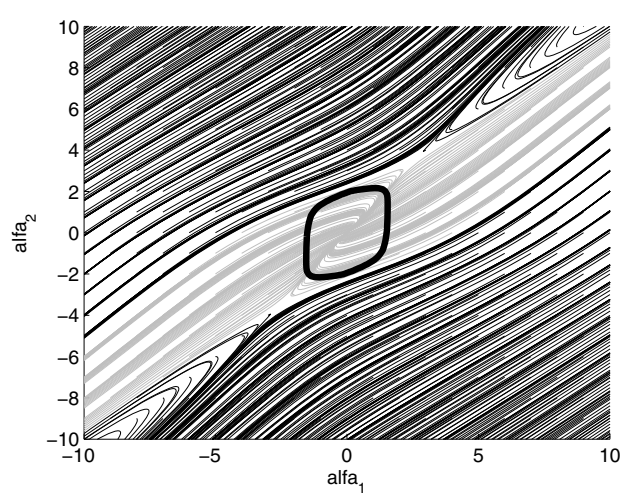

Fig. 4. Region of attraction set $V_{0}=1$.

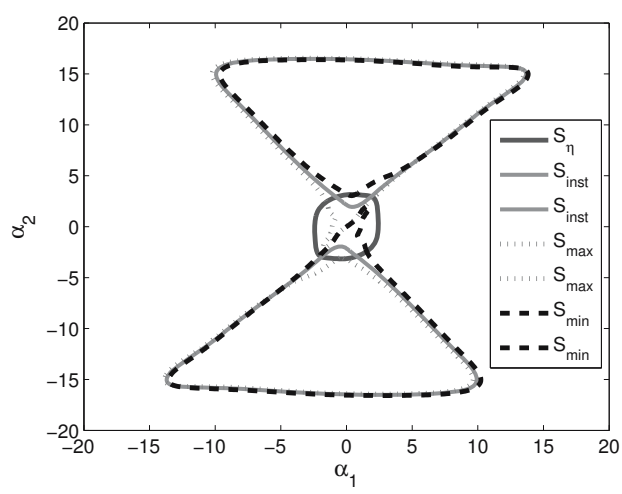

Fig. 5. Stability regions of the controlled system.

model parameters (1) are listed in Table 11 Two control actuation limits are compared, such as differential braking $M_{b r}$ and steering $\delta$. Since the steering model depends on $\dot{\delta}$, three scenarios are included in this section: braking, steering with $\nu=30$ and steering with $\nu=1$. The value $\nu=30$ is related to a fast actuation, e.g., active steering actuation, while $\nu=1$ represents low actuation, e.g., driver actuation. The actuation limits are $\left|\delta_{\max }\right|=12^{\circ}$ and $\left|M_{b r, \max }\right|=15000 \mathrm{Nm}(\mu=1)$.

The actuation limits at $\mu=1$ are illustrated in Fig. 6(a), The limits are calculated at four different velocities between $v=10 \mathrm{~m} / \mathrm{s}$ and $v=40 \mathrm{~m} / \mathrm{s}$. The results show that the actuation limits differ significantly in the three scenarios. In all cases, the sizes of the maximum controlled invariant sets are reduced, which is induced by the increase in unstable regions $S_{\text {unst }}$. This means that the stability of the vehicle can be guaranteed in a smaller region at higher velocity and peak-bounded control input. The increase in the velocity reduces the regions where stability is guaranteed. Note that the reduction of the sets could only be balanced with the increase of the actuation limits $u_{\max }$. However, it has limits on the control actuations. The enhancement of $\left|M_{b r, \max }\right|$ modifies the longitudinal dynamics with the 
Table 1. Data of tire models.

\begin{tabular}{|c||cc|}
\hline \multicolumn{1}{|c||}{$c_{i}$} & \multicolumn{2}{c|}{$\mu=1$} \\
& front & rear \\
\hline \hline$c_{1}$ & $1.9974 \cdot 10^{3}$ & $1.5594 \cdot 10^{3}$ \\
$c_{2}$ & $1.6601 \cdot 10^{-3}$ & $-1.1455 \cdot 10^{-2}$ \\
$c_{3}$ & $-5.0667 \cdot 10^{1}$ & $-3.9619 \cdot 10^{1}$ \\
$c_{4}$ & $2.6228 \cdot 10^{-3}$ & $4.4019 \cdot 10^{-3}$ \\
$c_{5}$ & $6.5909 \cdot 10^{-1}$ & $5.1212 \cdot 10^{-1}$ \\
$c_{6}$ & $6.5934 \cdot 10^{-6}$ & $3.5811 \cdot 10^{-5}$ \\
$c_{7}$ & $-3.8106 \cdot 10^{-3}$ & $-2.9443 \cdot 10^{-3}$ \\
$c_{8}$ & $-3.6916 \cdot 10^{-7}$ & $-9.0387 \cdot 10^{-7}$ \\
$c_{9}$ & $7.8771 \cdot 10^{-6}$ & $6.0612 \cdot 10^{-6}$ \\
$c_{10}$ & $1.3622 \cdot 10^{-9}$ & $3.1025 \cdot 10^{-9}$ \\
\hline \hline$c_{i}$ & \multicolumn{3}{|r}{$\mu=0.4$} \\
$c_{i}$ & front & rear \\
\hline \hline$c_{1}$ & $8.9427 \cdot 10^{2}$ & $8.8444 \cdot 10^{2}$ \\
$c_{2}$ & $-3.0717 \cdot 10^{-3}$ & $-3.7746 \cdot 10^{-3}$ \\
$c_{3}$ & $-2.9995 \cdot 10^{1}$ & $-3.4215 \cdot 10^{1}$ \\
$c_{4}$ & $1.6903 \cdot 10^{-3}$ & $2.6341 \cdot 10^{-3}$ \\
$c_{5}$ & $5.4779 \cdot 10^{-1}$ & $5.4459 \cdot 10^{-1}$ \\
$c_{6}$ & $3.3324 \cdot 10^{-5}$ & $3.1365 \cdot 10^{-3}$ \\
$c_{7}$ & $-4.5595 \cdot 10^{-3}$ & $-3.4066 \cdot 10^{-3}$ \\
$c_{8}$ & $-1.0084 \cdot 10^{-6}$ & $-3.9862 \cdot 10^{-5}$ \\
$c_{9}$ & $1.3562 \cdot 10^{-5}$ & $7.0489 \cdot 10^{-6}$ \\
$c_{10}$ & $4.8295 \cdot 10^{-9}$ & $1.2344 \cdot 10^{-7}$ \\
\hline & & \\
& & \\
\hline
\end{tabular}

increase in the longitudinal slips, and it is disadvantageous for the lateral force characteristics. Moreover, the increase in $\left|\delta_{\max }\right|$ influences $\alpha_{1}$ significantly, and the tire force characteristics have a decrease in high lateral slips. It can also be established that, at a low velocity, control actuation selection is irrelevant for the aspect of the maximum controlled invariant sets.

The computation results show that the maximum controlled invariant sets of steering significantly depend on $\nu$. The small variability in $\delta$ results in large sets, while a large variability in $\delta$ is connected to the increase in unstable regions. According to the results, at high velocity it is particularly important to actuate smoothly, without sudden transitions. Differential braking has a fixed region in the $\alpha_{1}-\alpha_{2}$ state-space depending on the velocity. Therefore, braking actuation is of importance when fast intervention is necessary. Since the maximum controlled invariant sets of steering can perceptibly be reduced at fast actuation, braking can be an appropriate solution to actuate in a short time and guarantee stability in a large region. In this case, the second scenario is not satisfactory, since it requires longer actuation time.

In Fig. 6(b), computation of the maximum controlled invariant sets for the tire-road adhesion coefficient $\mu=$ 0.4 is illustrated. In the case of differential braking, the intervention limit is reduced to $\left|M_{b r, \max }\right|=6000$ $\mathrm{Nm}$, because the maximum longitudinal braking force is

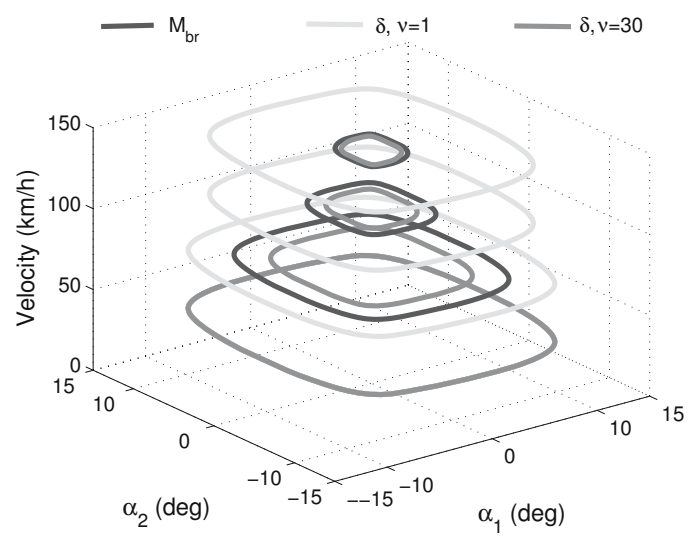

(a)

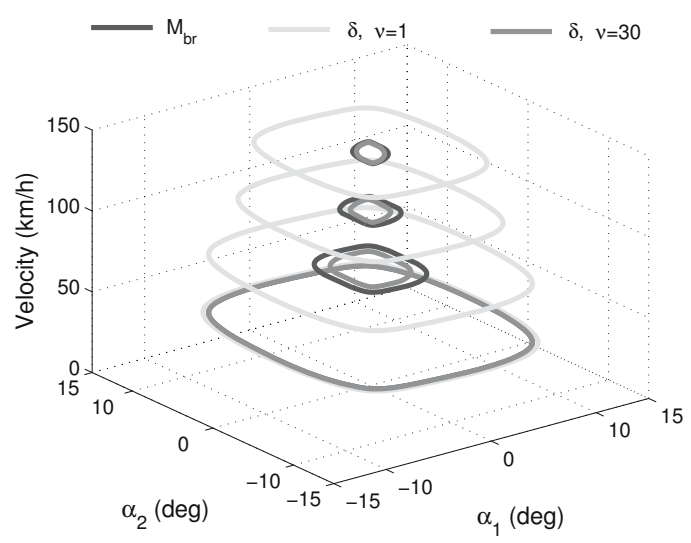

(b)

Fig. 6. Analysis of actuation limits: adhesion coefficient $\mu=1$ (a), adhesion coefficient $\mu=0.4$ (b).

reduced at low $\mu$. The reduction of adhesion coefficient $\mu$ induced the increase in regions $S_{\text {unst }}$, so all of the maximum controlled invariant sets are reduced. The velocity dependence of the regions is the same as proposed in the previous case: at higher velocity, a smaller region can be stabilized by the actuation. Parameter $\nu$ has the same influence on the maximum regions as in the $\mu=1$ scenario: an increase in the actuation speed reduces stable regions. Differential braking can be the appropriate solution to short time intervention.

\section{Control strategy based on the maximum controlled invariant sets}

In the section, a control strategy based on the maximum controlled invariant sets is presented. The intervention of the actuators assists the driver in performing vehicle maneuvers. The aim of the control is to guarantee the stability of the vehicle based on the SOS analysis.

The basic idea of the control strategy is the following. The stability of the polynomial system is analyzed using 
the control Lyapunov function. With this function and bounded control input, the stable and unstable regions of the system are computed (see, e.g., Fig. 5). There are some regions which are originally not stable, but an appropriate control input can stabilize them. The regions can be determined and, by the monitoring of $\alpha_{1}, \alpha_{2}$, the sufficient control input is applied.

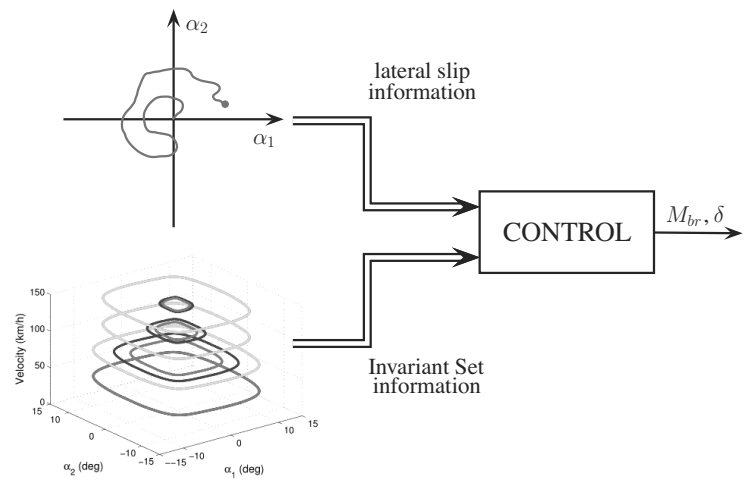

Fig. 7. Scheme of the control strategy.

The goal of the simulation scenario is to show an application possibility of the results of the analysis. As an illustration, the computed maximum control inputs are applied to the lateral dynamics of the vehicle. Thus, a bang-bang control rule is applied either to the steering or braking control. The invariant sets of the method are computed off-line and built in the vehicle control system. In the control application, it is necessary to compute several sets, which belong to different velocities and adhesion coefficients. The proposed algorithm requires the measurement of the velocity and the estimation of $\alpha_{1}$, $\alpha_{2}$ and $\mu$. The estimation of the adhesion coefficient $\mu$ in the paper is based on the time-varying Kalman filter method (see Gustafsson, 1997). An algorithm to estimate $\alpha_{1}$ and $\alpha_{2}$ is proposed by Grip et al. (2008). In the control algorithm, the decision about the intervention is based on the current lateral slips using the calculated maximum controlled invariant set at given $v$ and $\mu$.

The illustration of the strategy is shown in Fig. 7 . The estimation of lateral slip angles and the maximum controlled invariant sets are necessary for the control signals. The control values are in the set $u=$ $\left\{-u_{\max } ; 0 ; u_{\max }\right\}$. Since the determination of the maximum controlled invariant sets requires a great deal of computational effort, an offline computation of the sets is recommended. It is necessary to determine the unstable regions and the appropriate control input for stabilization.

6.1. Illustration example. In the following, the control application of a maximum controlled invariant sets through a simulation scenario in the CarSim simulation environment is proposed. CarSim provided a vehicle dynamic simulator, together with the vehicle parameters. The performing of the simulation is based on Matlab/Simulink, in which the CarSim model is embedded, together with the designed control algorithm. The measured signals for the controller are provided by CarSim. The control algorithm computes the actual intervention $M_{b r}$ from the signals, and it is transformed to braking pressures, as found in the work of Németh and Gáspár (2011). The braking pressures on the inputs of the CarSim model are actuated.

In the scenario, the driver must avoid an obstacle on the $\operatorname{road}(\mu=1)$. Thus, the path of the vehicle is suddenly modified, which creates a critical situation. In this scenario, differential braking is applied to assist the driver in performing the maneuver. Two vehicles are compared in the simulation: the first vehicle uses the proposed control strategy, while the second vehicle is uncontrolled.

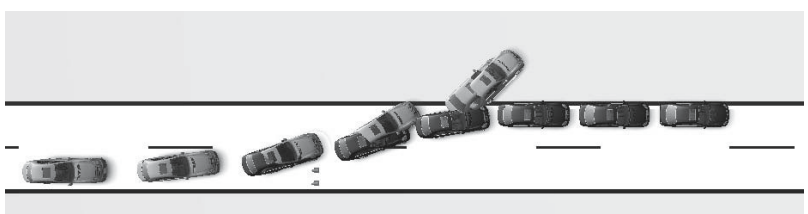

Fig. 8. Paths of the vehicles.

The paths of the vehicles are illustrated in Fig. 8, Due to the obstacle on the road, the driver changes lanes. In the maneuver, the steering angle (Fig. 9(a) and the velocity of the vehicle (Fig. 9(b) are modified. Simultaneous braking and sudden steering may be dangerous, because the lateral slip values of the tire change significantly. The $\alpha_{1}, \alpha_{2}$ state trajectories of the vehicles and the maximum controlled invariant sets at different velocities are shown in Fig. 9(d), The lateral slip values of the controlled vehicle converge back to zero during the differential braking actuation $M_{b r}$ (Fig. 9(c)], while the states of the uncontrolled vehicle increase and move to the unstable region. Thus, the control strategy presented above is able to stabilize the vehicle. In the simulation, the motions of the vehicles (Fig. 8) illustrate the consequences of the different state trajectories: the uncontrolled vehicle (gray) rolls off the road, while the controlled vehicle (black) is stabilized and road holding is guaranteed.

The simulation results show that the maximum controlled invariant sets can be used to stabilize the vehicle. The intervention based on the analysis is able to prevent the vehicle from leaving the road.

\section{Conclusions}

In the paper, the stabilization regions of the steering and braking systems are examined in order to analyze 


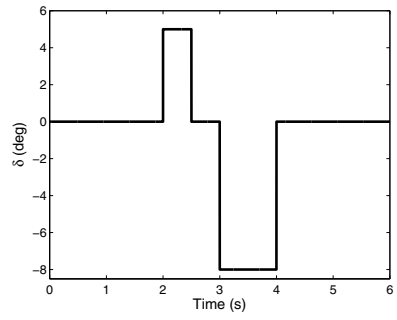

(a)

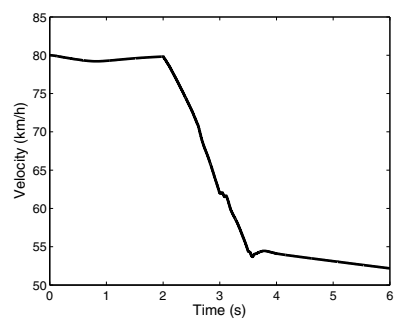

(b)

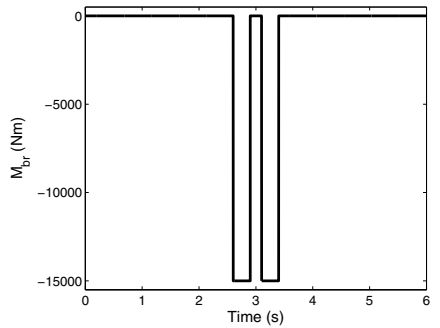

(c)

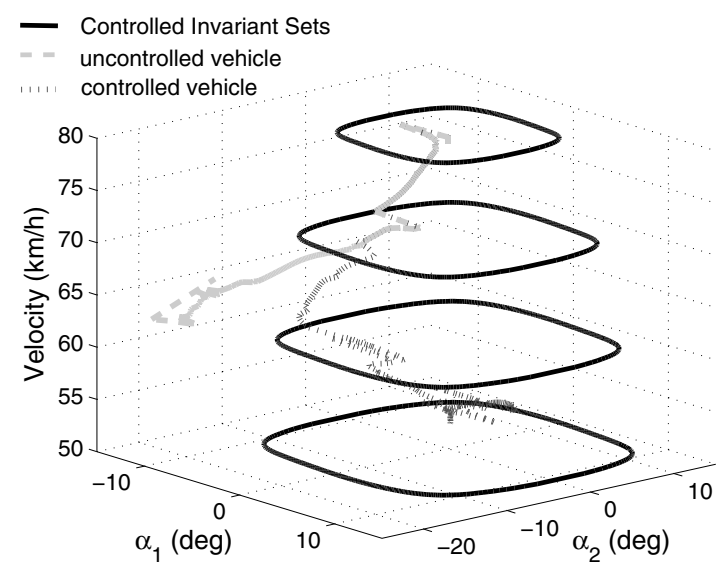

(d)

Fig. 9. Simulation scenario: steering angle (a), velocity of vehicle (b), control input (c), state trajectories (d).

their abilities to intervene in the entire vehicle system. A nonlinear polynomial SOS programming method is applied to calculate the shape of the controlled invariant sets of control actuations. The aim of the analysis is to provide a theoretical basis for the coordination of vehicle control interventions. The method is illustrated through the influence of the steering and brake control systems at various velocities and road conditions.

The presented computation scenarios show that the velocity and the adhesion coefficient have a significant influence on the maximum invariant sets. Thus, different vehicle dynamic regions can be reached and stabilized by differential braking and steering.

\section{Acknowledgment}

The research was supported by the National Research, Development and Innovation Fund through the project SEPPAC: Safety and Economic Platform for Partially Automated Commercial Vehicles (VKSZ 14-1-2015-0125). This paper was partially supported by the János Bolyai Research Scholarship of the Hungarian Academy of Sciences.

\section{References}

Beal, C.E. and Gerdes, J.C. (2013). Model predictive control for vehicle stabilization at the limits of handling, IEEE Transactions on Control Systems Technology 21(4): 1258-1269.

Cairano, S., Tseng, H.E., Bernardini, D. and Bemporad, A. (2013). Vehicle yaw stability control by coordinated active front steering and differential braking in the tire sideslip angles domain, IEEE Transactions on Control Systems Technology 21(4): 1236-1248.

Carvalho, A., Palmieri, G., Tseng, H., Glielmo, L. and Borrelli, F. (2013). Robust vehicle stability control with an uncertain driver model, European Control Conference, Zurich, Switzerland, pp. 440-445.

de Wit, C.C., Olsson, H., Astrom, K.J. and Lischinsky, P. (1995). A new model for control of systems with friction, IEEE Transactions on Automatic Control 40(3): 419-425.

Grip, H., Imsland, L., Johansen, T., Fossen, T., Kalkkuhl, J. and Suissa, A. (2008). Nonlinear vehicle side-slip estimation with friction adaptation, Automatica 44(11): 611-622.

Gustafsson, F. (1997). Slip-based tire-road friction estimation, Automatica 33(6): 1087-1099.

Jarvis-Wloszek, Z. (2003). Lyapunov Based Analysis and Controller Synthesis for Polynomial Systems using Sumof-Squares Optimization, Ph.D. Thesis, University of California, Berkeley, CA.

Jarvis-Wloszek, Z., Feeley, R., Tan, W., Sun, K. and Packard, A. (2003). Some controls applications of sum of squares programming, 42nd IEEE Conference on Decision and Control, Maui, HI, USA, Vol. 5, pp. 4676-4681.

Jianyong, W., Houjun, T., Shaoyuan, L. and Wan, F. (2007). Improvement of vehicle handling and stability by integrated control of four wheel steering and direct yaw moment, 26th Chinese Control Conference, Zhangjiajie, China, pp. 730-735. 
Khelassi, A., Theilliol, D. and Weber, P. (2011). Reconfigurability analysis for reliable fault-tolerant control design, International Journal of Applied Mathematics and Computer Science 21(3): 431-439, DOI: 10.2478/v10006-011-0032-z.

Kiencke, U. and Nielsen, L. (2000). Automotive Control Systems for Engine, Driveline and Vehicle, Springer, New York, NY.

Kim, D., Peng, H., Bai, S. and Maguire, J. (2007). Control of integrated powertrain with electronic throttle and automatic transmission, IEEE Transactions on Control Systems Technology 15(3): 474-482.

Korda, M., Henrion, D. and Jones, C.N. (2013). Convex computation of the maximum controlled invariant set for discrete-time polynomial control systems, Conference on Decision and Control, Firenze, Italy, pp. 7107-7112.

Kritayakirana, K. and Gerdes, J. (2012a). Using the centre of percussion to design a steering controller for an autonomous race car, Vehicle System Dynamics 50(Supp1): 33-51.

Kritayakirana, K. and Gerdes, J.C. (2012b). Autonomous vehicle control at the limits of handling, International Journal of Vehicle Autonomous Systems 10(4): 271-296.

Lasserre, J.B. (2007). Sum of squares approximation of nonnegative polynomials, SIAM Journal on Optimization 49(4): 651-669.

Löfberg, J. (2009). Pre- and post-processing sum-of-squares programs in practice, IEEE Trans. on Automatic Control 54(5): 1007-1011.

Lu, J. and Filev, D. (2009). Multi-loop interactive control motivated by driver-in-the-loop vehicle dynamics controls: The framework, Conference on Decision and Control, Shanghai, China, pp. 3569-3574.

Mastinu, G., Babbel, E., Lugner, P., Margolis, D., Mittermayr P. and Richter, B. (1994). Integrated controls of lateral vehicle dynamics, Vehicle System Dynamics 23(Supp1): 358-377.

Németh, B. and Gáspár, P. (2011). Design of actuator interventions in the trajectory tracking for road vehicles, Conference on Decision and Control, Orlando, FL, USA, pp. 7434-7439.

Németh, B. and Gáspár, P. (2013). Analysis of vehicle actuators based on reachable sets, European Control Conference, Zurich, Switzerland, pp. 3137-3142.

Ono, E., Hattori, Y., Muragishi, Y. and Koibuchi, K. (2006). Vehicle dynamics integrated control for four-wheel-distributed steering and four-wheel-distributed traction/braking systems, Vehicle System Dynamics 44(2): 139-151.

Pacejka, H.B. (2004). Tyre and Vehicle Dynamics, Elsevier Butterworth-Heinemann, Oxford.

Palmieri, G., Barc, M., Glielmo, L., Tseng, E.H. and Borrelli, F. (2011). Robust vehicle lateral stabilization via set-based methods for uncertain piecewise affine systems: Experimental results,50th IEEE Conference on Decision and Control, Orlando, FL, USA, pp. 3252-3257.
Palmieri, G., Baric, M., Glielmo, L. and Borrelli, F. (2012) Robust vehicle lateral stabilisation via set-based methods for uncertain piecewise affine systems, Vehicle System Dynamics 50(6): 861-882.

Papachristodoulou, A. and Prajna, S. (2005). Analysis of non-polynomial systems using the sum of squares decomposition, in D. Henrion and A. Garulli (Eds.), Positive Polynomials in Control, Springer-Verlag, Berlin/Heidelberg, pp. 23-43.

Parrilo, P. (2003). Semidefinite programming relaxations for semialgebraic problems, Mathematical Programming $B$ 96(2): 293-320.

Poussot-Vassal, C., Sename, O., Dugard, L., Gáspár, P., Szabó, Z. and Bokor, J. (2008). A new semi-active suspension control strategy through LPV technique, Control Engineering Practice 16(12): 1519-1534.

Prajna, S., Papachristodoulou, A. and Wu., F. (2004). Nonlinear control synthesis by sum of squares optimization: A Lyapunov-based approach, 5th IEEE Asian Control Conference, Melbourne, Australia, Vol. 1, pp. 157-165.

Sadri, S. and Wu, C. (2013). Stability analysis of a nonlinear vehicle model in plane motion using the concept of Lyapunov exponents, Vehicle System Dynamics 51(6): 906-924.

Scherer, C.W. and Hol, C.W.J. (2006). Matrix sum-of-squares relaxations for robust semi-definite programs, Mathematical Programming 107(1): 189-211.

Sontag, E.D. (1989). A “universal” construction of Artstein's theorem on nonlinear stabilization, Systems \& Control Letters 13(2): 117-123.

Summers, E., Chakraborty, A., Tan, W., Topcu, U., Seiler, P., Balas, G. and Packard, A. (2003). Quantitative local $l_{2}$-gain and reachability analysis for nonlinear systems, International Journal of Robust and Nonlinear Control 23(10): 1115-1135.

Tan, W. and Packard, A. (2008). Stability region analysis using polynomial and composite polynomial Lyapunov functions and sum-of-squares programming, IEEE Transactions on Automatic Control 53(2): 565-571.

Topcu, U. and Packard, A. (2009). Local robust performance analysis for nonlinear dynamical systems, American Control Conference, St. Louis, MO, USA, pp. 784-789.

Yetendje, A., Seron, M.M. and De Doná, J.D. (2012). Robust multisensor fault tolerant model-following MPC design for constrained systems, International Journal of Applied Mathematics and Computer Science 22(1): 211-223, DOI: 10.2478/v10006-012-0016-7.

Yu, F., Li, D. and Crolla, D. (2008). Integrated vehicle dynamics control: State-of-the art review, IEEE Vehicle Power and Propulsion Conference, Harbin, China, pp. 1-6.

Zhang, S., Zhang, T. and Zhou, S. (2009). Vehicle stability control strategy based on active torque distribution and differential braking, Conference on Measuring Technology and Mechatronics Automation, Zhangjiajie, Hunan, China, pp. 922-925. 


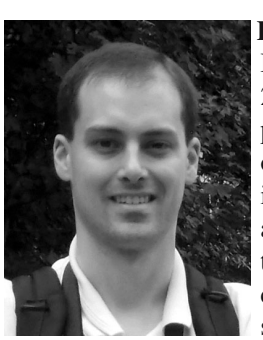

Balázs Németh has been a research fellow of the Institute for Computer Science and Control since 2007. He received his Ph.D. degree in transportation sciences from the Budapest University of Technology and Economics in 2013. His most important research areas are nonlinear analysis and synthesis of integrated vehicle control systems, driver assistance systems, energy-optimal control of road vehicles, and variable geometry suspension control.

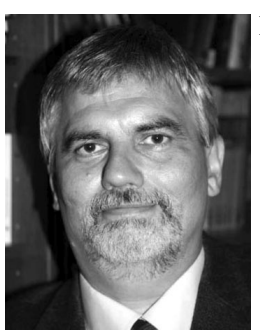

Péter Gáspár has been a research advisor at the Systems and Control Laboratory (SCL), Computer and Automation Research Institute, since 1990. He is the head of the Vehicle Dynamics and Control Research Group within the SCL. He is also a full professor at the Control and Transport Automation Department, Budapest University of Technology and Economics. He is a member of the IFAC Technical Committees on both Automotive Control and Transportation Systems. His research interests include linear and nonlinear systems, robust control, multi-objective control, system identification and identification for control. His research and industrial works have involved mechanical systems, vehicle structures and vehicle control.

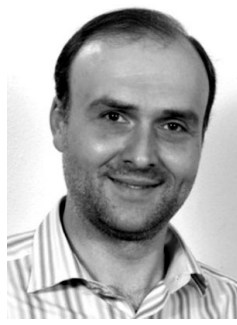

Tamás Péni is a senior research fellow at the Institute for Computer Science and Control. His research interest focuses mainly on the analysis and control of linear time invariant and parametervarying systems. As a member of the Theory of Dynamical Systems Research Group, he is working on the development of robust and constrained control methods, as well as on fault detection and accommodation algorithms. These methods are developed mainly for aerospace applications.

Received: 4 November 2014

Revised: 27 May 2015

Re-revised: 6 August 2015 